\title{
Physiotherapy and Anterior Repositioning Splint in the Treatment of Disk Displacement with Reduction - A Randomized Controlled Trial
}

\author{
Irfan Adil Majid1 ${ }^{1}$, Mubeen², Fazeena Karimalakuzhiyil Alikutty ${ }^{3}$ \\ 1Department of Oral Basic Clinical Sciences, Ibn Sina National College for Medical \\ Studies, Jeddah, Saudi Arabia. ${ }^{2}$ Government Dental College and Research Institute, \\ Bangalore, India. ${ }^{3}$ Ibn Sina National College for Medical Studies, Jeddah, Saudi Arabia.
}

\section{ABSTRACT}

\section{BACKGROUND}

Treatment recommendations for symptomatic disk displacement with reduction (DDwR) range from conservative to aggressive and irreversible treatment. This study investigated and compared the effectiveness of self-care in combination with physiotherapy and anterior repositioning splint in treating patients with DDwR.

\section{METHODS}

This single (assessor) blinded randomized clinical trial comprised of three groups with 63 study subjects in each. Group I (SC/control group) received self-care instructions (jaw relaxation in the form of diet of soft food, avoiding gum chewing and reduction of oral parafunctional habits, thermal packs, jaw exercises and correcting bad posture). Group II (PT group) was advised US therapy 0.25 watts / $\mathrm{cm}^{2}$ pulsed at two - 1, 2 - 3 minutes / application, 3 - 4 times a week for four weeks. Group III (ARS group) received clear and hard acrylic Anterior Repositioning Splint set to the upper teeth; additionally the PT and ARS groups were also advised similar TMD self-care procedures as SC group. The outcomes [TMJ pain, Tenderness Muscle of Mastication (TMoM), Comfortable Mouth Opening (CMO), Maximum Mouth Opening (MMO) and TM joint clicking] were measured at weekly (for 4 weeks) intervals for SC and PT group and monthly (for 3 months) interval for ASR group to measure short term benefits. While the long term benefits of all 3 groups were measured post treatment after 16 weeks post trial commencement. One-way ANOVA (for continuous data measures) was used to compare the treatment outcomes of all the 3 groups, $\chi^{2}$ test was used to compare treatment proportions for categorical measures followed by the post hoc Tukey test. The level of significance for all the tests was fixed at $\mathrm{P} \leq 5$ ( $5 \%$ probability).

\section{RESULTS}

All the study groups showed significant improvement in joint pain and TMoM. While the TMJ clicking significantly reduced in the ARS group only. Both CMO and MMO improved in all three groups, but was maximum in the ARS group. Post treatment (four months) follow-up showed a significant relapse of pain and mouth opening in SC and PT groups, which was comparatively less in the ARS group with regard to joint pain, muscle tenderness, mouth opening, and clicking.

\section{CONCLUSIONS}

Standalone all three treatment modalities were significantly effective in alleviating the signs and symptoms of DDwR. Equivalent improvement in the three groups implies encouraging clinical applications in terms of different treatment time, and cost involved to deliver them. Therefore, a comprehensive care of combination therapies rather than a monotherapy seems more suitable and practical choice to treat DDwR, especially, in view of the cost effectiveness and promptness of the treatment.

\section{KEY WORDS}

TMD, Disk Displacements with Reduction, TMJ Physiotherapy, Ultrasound Physiotherapy, Splint Therapy, Anterior Repositioning Splint
Corresponding Author:

Dr. Irfan Adil Majid,

Division of Oral Medicine and Radiology, Department of Oral Basic Clinical Sciences, Ibn Sina National College for Medical Studies, Jeddah, Saudi Arabia.

E-mail: drirfanadil@yahoo.com

DOI: $10.14260 / \mathrm{jemds} / 2020 / 860$

How to Cite This Article:

Majid IA, Mubeen, Alikutty FK. Physiotherapy and anterior repositioning splint in the treatment of disk displacement with reduction-a randomized controlled trial. J Evolution Med Dent Sci 2020;9(52): 3926-3934, DOI

10.14260/jemds/2020/860

Submission 06-09-2020,

Peer Review 01-11-2020,

Acceptance 09-11-2020,

Published 28-12-2020.

Copyright (C) 2020 Irfan Adil Majid et al. This is an open access article distributed under Creative Commons Attribution License [Attribution 4.0 International (CC $B Y 4.0)]$ 


\section{BACKGROUND}

Disc Displacement with Reduction (DDwR) is a form of internal derangement of the temporomandibular joint (TMJ) wherein the articular disc is displaced anterior to the condylar head or sometimes may be displaced medially or laterally (with or without intermittent locking). ${ }^{1}$ DDwR can presents with pain and / or dysfunction, joint clicking may or may not be accompanied.1,2,3,4,5,6 Among many conservative and reversible (non-surgical) treatment approaches self-care [thermal packs (moist heat fermentation), jaw relaxation in the form of diet of soft food, avoiding gum chewing and reduction of oral parafunctional habits, jaw exercises correcting bad posture], anterior repositioning splints (AR splint) and physiotherapy in the form of ultrasound (US therapy) therapy are the most common non-surgical treatment options recommended to treat temporomandibular disorders (TMD) and DDwR.7,8,9

Although US therapy is commonly employed in the management of TMDs there is little published material reporting the comparative efficacy of different type of treatment currently available ${ }^{9}$ While several studies have investigated the effectiveness of physical therapy and splint therapy in patients with Disk Displacement without reduction (DDwoR), $7,8,9,10,11$ there is a relative deficiency of the effectiveness of these treatment modalities on DDwR. The Randomized Controlled Trials (RCT) have also failed to demonstrate a distinct positive effect of splints.12,13,14 Although a number of previous studies have demonstrated both, equal to ${ }^{12,15,16}$ or weaker ${ }^{17,18}$ outcomes in comparison with the other known treatment options employed in the management of TMDs. However, from the point of view of evidence-based practice, the direct comparison between selfcare, US therapy and AR splint therapy in the treatment of DDwR is limited due to the heterogeneity of the study subjects and the weak inclusion criteria. Thus leading to a limited scope in their application to determine the effectiveness to treat DDwR. The preceding reasons led us to conduct this RCT to address the aforementioned knowledge gaps with the primary aim to assess the effectiveness and compare self-care (SC) with Ultrasound Physiotherapy (US Therapy); SC with hard acrylic Anterior Repositioning Splints (ARS) therapy; and SC strategies alone. The secondary aim was to determine whether adding two types of reversible conservative therapies to selfcare offered any advantage in reducing the DDwR related signs and symptoms over self-care strategies alone.

\section{METHODS}

This was a single (assessor) blinded randomized clinical trial designed to test the efficacy of physiotherapy (PT), anterior repositioning splint (ARS) and self-care (SC) in the control of signs and symptoms of DDwR.

\section{Trial Design}

This study was a single (assessor) blinded RCT with three trial groups running in parallel. The study was conducted over period of twelve months from baseline (BL) to follow-up of outcome assessment.

\section{Setting and Participants}

The trial was conducted on subjects recruited form the outpatient clinics of Oral Medicine and Radiology department of a Teaching Hospital in Bangalore, India who fulfilled the following criteria:

\section{Inclusion Criteria}

Report of pain in pre-auricular region, in the last thirty days that worsened by functional activities, such as chewing and talking. Presence of disc displacement with reduction (IIa) and arthralgia (IIIa) according to the Research Diagnostic Criteria for Temporomandibular Disorders (RDC / TMD Axis I).19,20,21

\section{Exclusion Criteria}

Subjects diagnosed with any other RDC / TMD Axis I conditions (arthritis, disk displacement without reduction) and RDT / TMD axis II (psychological disorders). Individuals with a recent history of trauma to the face and / or neck area; with systemic diseases that can affect TMJ and TMJ surgery. Individuals with dental pain, with myofascial pain or osteoarthritis. Individuals undergoing active dental and or TMD management and individuals wearing full or partial dentures were excluded.

\section{The Study Sample}

The sample size of this study was calculated based on a priori sample size calculation method using G *Power software* package (version 3.1.4). Based on the following parameters: input effect size $\mathrm{f}=01, \alpha$ error $=0.05$, power $=0.8$, number of groups $=3$, number of measurements $=6$, the total sample size calculated was 43 subjects $(260 / 6=43.3)$ per group. However, we increased the number of study subjects to 63 subjects per group in case we lost them to follow-up.

\section{Trial Interventions}

All participants diagnosed with RDC / TMD axis I TMD were included, the eligible subjects completed a survey that included the Research Diagnostic Criteria for Temporomandibular Disorders (RDC / TMD) ${ }^{19}$ questionnaire, and additional questions about symptoms, treatment, and medical and behavioural status. ${ }^{20,21}$

Patients of three study groups were provided with selfcare instructions, then were randomly enrolled into one of the three study groups namely; Group I / self-care (SC) / control group, Group II / Ultrasound Physiotherapy (PT) group and Group III / Anterior Repositioning Splint (ARS) group.

Group I / Self-care (SC) / control group: The SC group received education about TMD self-care which included thermal packs (moist heat fermentation), jaw relaxation (soft food diet, avoiding gum chewing and reduction of oral parafunctional habits), jaw exercises correcting bad posture and one SOS nonsteroidal anti-inflammatory drugs (NSAIDs) in case of acute pain.

Group II / Ultrasound Physiotherapy (PT) group: the PT group was advised Ultrasound Physiotherapy (US therapy) by an experienced physiotherapist plus TMD self-care instructions.

The US therapy procedure: ultrasound of 0.25 watts / centimeter ${ }^{2}$ pulsed at 2 - 1 was applied for 2 - 3 minutes per 
visit 3 - 4 times a week for four weeks. Then remained untreated for the next 12 weeks [three months] and a final follow-up after four months (sixteen weeks) from BL. ${ }^{9}$

Group III / Anterior Repositioning Splint (ARS) group: advised AR splint plus TMD self-care instructions. The AR splint was fabricated with clear, self-curing hard acrylic resin that could be set to maxillary teeth to provide equal contact for all the lower teeth plus the self-care instructions.

Fabrication of AR splint: Jaw impressions of patients of ARS group were made to fabricate the anterior repositioned hard acrylic splint. The anterior bite position for the splint was determined by guiding the patient's mandible forward until the disc / discs are reduced and the patient could open and close the mouth without TMJ click. The anterior bite position was then indexed into the splint while fabricating it. The finished splint with protruded occlusion was delivered to the patient. The participants were advised to wear splint at nighttime for three months and two hours each day while awake throughout the three-month follow-up. Further, they were advised to discontinue wearing the splint after third month visit for four weeks (one month). ${ }^{7}$

\section{Trial outcomes}

During follow-up, the outcomes were measured by first having the subjects' complete self-reported questionnaire and then RDC / TMD clinical examination was conducted by the trained and calibrated post graduate students blinded to study group allocation. The treatment outcomes measured at BL and all the subsequent follow-up visits: 7,9

1. TMJ Pain (4 TMJ sites) and tenderness of muscle of mastication (TMoM) palpation (10 extra oral, 4 intraoral) on Numerical Rating Scale (NRS)

2. Mouth opening: Comfortable Mouth Opening (CMO), Maximum Mouth Opening (MMO).

\section{TMJ clicking.}

Perception of pain and tenderness of TMJ and muscle of mastication and TMJ clicking was recorded by the participants on a Numerical Rating Scale (NRS) that contained scores ranging from 0 to 10 , mouth opening (both CMO and MMO) was measured as the distance between the upper and lower incisor using a calliper.

\section{Follow-Up}

We instructed all the study participants to return to the clinic for follow-up and to contact the study coordinator if any problem was encountered between the follow-up visits and during the course of trial. In case the pain symptoms worsened while undergoing the trial the patients were advised to discontinue the treatment (US therapy / use of AR splint) and use the analgesic that was prescribed. The AR splint group patients were advised to bring their splints to clinic visits to check for any discrepancies if developed while in use. The patients of SC and PT groups were recalled at weekly intervals for four weeks and ARS group was recalled after one week for post initial insertion evaluation of splint to make minor adjustments in the splint if necessary, thereafter, the patients were recalled for follow-up at the end of each month for three months. A final post-treatment follow-up of all three groups was carried out at the end of 16 weeks after BL (four months).

\section{Trial Procedure}

The procedure of enrolment, intervention and assessment are shown in CONSORT flow diagram in figure 1.

\section{Randomisation}

Clinicians of the department of oral medicine and radiology attending the out-patient clinics experienced in the use of research and diagnostic criteria for temporomandibular disorders performed standardized RDC / TMD Axis I clinical examinations on the subjects at the initial visit to recruit subjects to the clinical trial. The study provider / study coordinator (statistician) stratified the participants and block randomised (block of six, nine or 12 subjects) them by computer generated random numbers to equalize the number of participants in the three study groups. The computer generated random numbers were informed to the principal investigator (not directly involved in data collection) who assigned the participants to the intervention groups to minimise contamination of study group. Concealment of allocation was maintained up to the main statistical analysis and fixing of the data.

\section{Blinding}

The participants and care providers (physiotherapist and clinicians fabricating and delivering the anterior repositioning splint) could not be blinded in this study owing to the nature of the intervention. The patients knew to which intervention group they were allocated (after reading the information sheet, referral letter and the consent form), however, the outcome assessors were blinded to the intervention group.

\section{Ethical Approval}

The proposed study design was approved by the institutional review board and all the study participants signed an informed consent to participate in the study in accordance with human subjects' research ethics requirement of Helsinki.

\section{Data Collection}

We collected the study data as detailed above at BL, week one, two, three, four and 16 weeks from BL for SC and PT groups [9] and month one, two, three and four (16 weeks from $\mathrm{BL}$ ) for the ARS group patients. ${ }^{7}$

\section{Study Hypotheses}

We formed two hypotheses -

1. Hypothesis 1 (short-term improvement): at four weeks post-treatment, the subjects in the PT and ARS groups would demonstrate equivalent levels of short-term improvement in (self-reported pain and in clinical measures such as range of motion, tenderness of muscle of mastication to palpation and reduction in $\mathrm{TM}$ ] clicking) outcomes measured and, the subjects of SC group would show less short-term improvement compared to PT and ARS groups.

2. Hypothesis 2 (long-term improvement): At the 16 weeks (4 month from BL) follow-up visits, subjects of all three (SC, PT and ARS) groups would show equivalent 
improvement in DDwR related pain and other clinical and self-report measures.

\section{Data Analysis}

The data was analysed using IBM SPSS Statistics Version 22, we used one-way ANOVA to compare the mean scores for the treatment groups at follow-up for continuous data measures, while $\chi 2$ test was used to compare treatment proportions for categorical measures followed by post hoc Tukey test. In all the tests we considered $(p \leq 5)$ probability level of $5 \%$ to be significant.

\section{RESULTS}

Although we collected data at four different point of time while the trial was on, we are reporting data that is most relevant to our study hypotheses. The "short-term" outcomes / end of treatment (V3) four-weeks from the BL for SC and PT group and moth three from the BL for ARS group. The "long-term" outcome / follow-up (V4) is reported as sixteen-weeks from BL for all three groups, because we found week one and two data to be equivalent to BL and the week three's data was equivalent to V3. We included the data of all the randomised study subjects, even of those lost to follow-up (the participant's last TMD values were extended to fill in the postassessment time points) in line with the principle of intent to treat analysis.

\section{Demographics}

Table 1 shows the demographics and BL data of the three treatment groups, through our analysis we did not find any significant difference in the treatment groups at the BL. Treatment outcomes of 189 participants was analysed at the end of the trail; Group SC (Group I, $\mathrm{n}=63$ ), Group PT (Group II, $n=63$ ), Group ARS (Group III, $n=63$ ). The study population consisted of 73 males and 116 female participants, the age of the participants ranged from 21 - 49 years.

\section{Clinical Examination Findings}

The observations and results of the study were as follows:

1. TMJ disc displacement with reduction showed a highest prevalence in $2^{\text {nd }}$ to $4^{\text {th }}$ decades of life.

2. After treatment, the subjects in all the study groups showed improvement of joint pain and muscle tenderness.

3. There was a significant improvement in mouth opening in all three groups, but was maximum in ARS group.

4. Significant amount of reduction in TMJ clicking was noticed only in ARS group (both at V3 and V4).

5. There was significant relapse of pain and mouth opening in SC and PT groups at final follow-up after treatment (V4).

6. Less significant relapse (at V4) was observed in ARS group with regard to joint pain, muscle tenderness, mouth opening, and clicking.

Pain in the TM joint on Numerical Rating Scale (NRS);

Figure 2 shows the self-reported TM joint pain on NRS measuring one to ten. At $\mathrm{BL}$, the average TMJ pain was comparable across groups $(\mathrm{SC}=6.2, \mathrm{PT}=6.10, \mathrm{ARS}=6.33 ; \mathrm{P}=$ 0.81 ). The average score decreased significantly from a mean $( \pm$ SD) of $6.28( \pm 1.9)$ at BL to $1.19( \pm 2.3)$ at four weeks and $1.42( \pm 2.4)$ at 16 -weeks follow-up $(\mathrm{p}<.0001)$. While, pain scores decreased considerably across the three study groups, but one ANOVA did not show any significant difference in the pain reduction between the groups ( $\mathrm{p}$ value of $\mathrm{V} 3=0.84$ and V4 $=0.56$ ) Table 2 .

Tenderness of Muscle of Mastication to palpation (TMoM) (Figure 3): Although we observed a considerable reduction in TMoMto palpation of ten extra and four intraoral muscular sites within the three treatment groups. However, significant difference was not observed in reduction of TMoM to palpation between the treatment groups either in the short term or long term follow-up (V3: $p=0.75, V 4: p=0.72$ ).

Mouth opening: Comfortable Mouth Opening (CMO), Maximum Mouth Opening (MMO): We found significant increase in both CMO and MMO with in all the three treatment groups from $\mathrm{BL}$ to $\mathrm{V} 3$ ( $\mathrm{p}=0.01$ and $\mathrm{p}=0.02$ respectively) (Figure 4 and 5). However, only MMO showed significant improvement in V4 ( $p=0.04)$, but not CMO ( $p=0.07)$.

\section{TMJ Clicking}

The joint sounds decreased (Figure 6) significantly in only the ARS group by the end of the clinical trial (short term improvement) that was sustained even after the splint usage was discontinued (long term improvement). Although, PT group showed a marginal reduction in joint sounds (Table 2) yet, this improvement was not statistically significant.

\section{Compliance with Treatment and Study Dropouts}

A total of 189 patients were recruited in to our study, these study subjects reported good compliance with the treatment recommended across all the three groups. They reported almost uniform compliance with the self-care instructions given to them at the start of the trail, however, a modest difference was found in the later stages of SC group. No subjects reported any serious adverse effects with the treatment administered, there were no reports of any untoward incidents or complications with ultrasound physiotherapy or AR splint therapy. There were no significant occlusal changes identified, either by self-report or by clinical evaluation using full-arch articulating paper in AR splint users.

Among the 189 study subjects 36 (19.04\%) patients were lost to follow-up by the end of the clinical trial. We observed a high rate of patient retention in ARS group (90.47\%) followed by PT group (80.95\%) and was least in SC group (71.42\%). The ongoing treatment in PT and ARS groups (to keep-up with the treatment) could have been the reason for the high retention rate. Likewise no treatment (self-care only) may have led to poor adherence to the follow-up and high drop-out rate. Follow-up rates were similar among groups while the trail was underway, but they differed among ARS groups at 16 weeks ( $\mathrm{p}=.004, \chi^{2}$ test) post treatment final follow-up (Figure 1). However, we observed no significant differences in the primary outcome measures or the demographic or clinical measures at BL, except that those who completed the study reported more prior health care visits for TMJ related pain. 


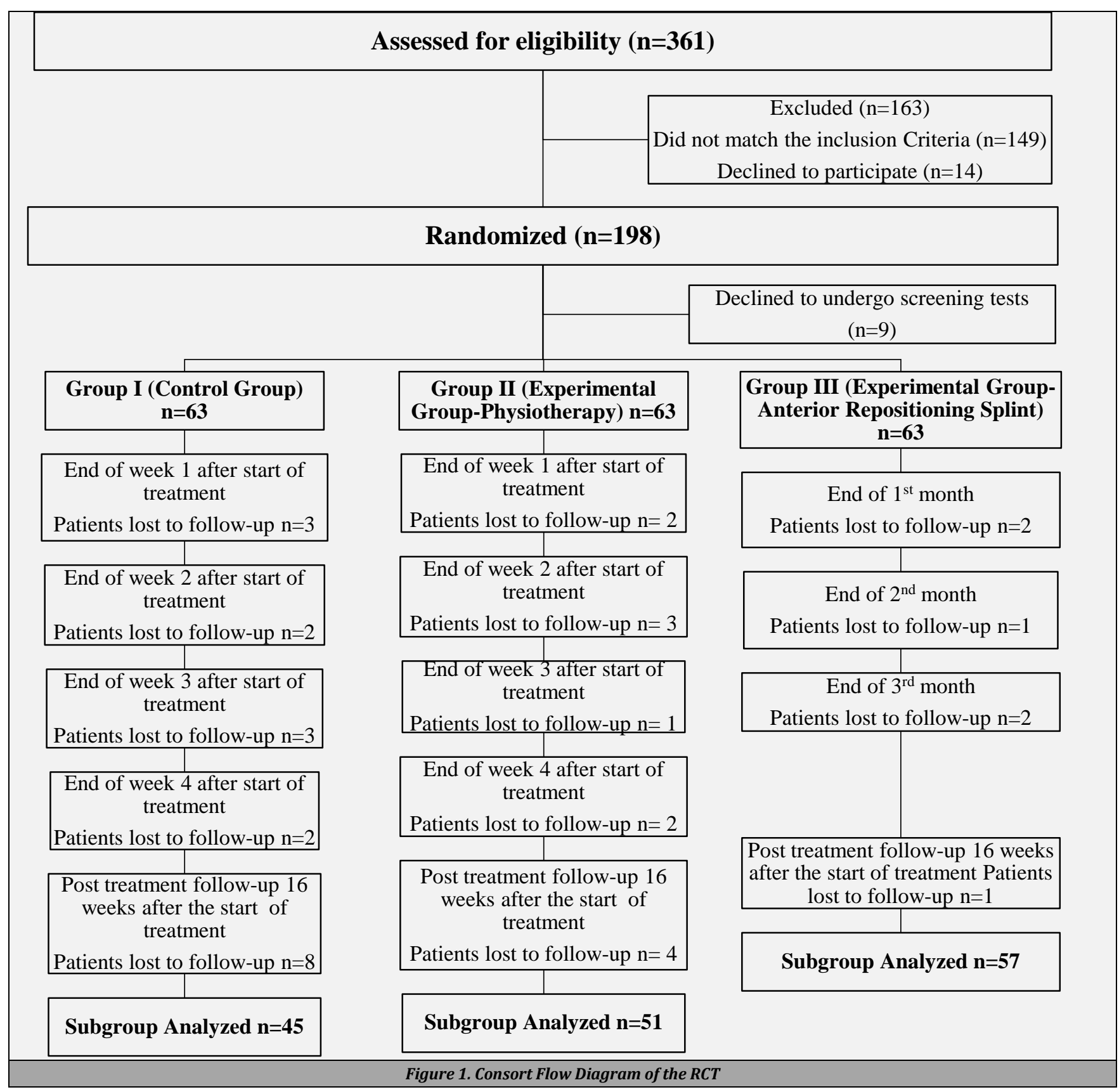

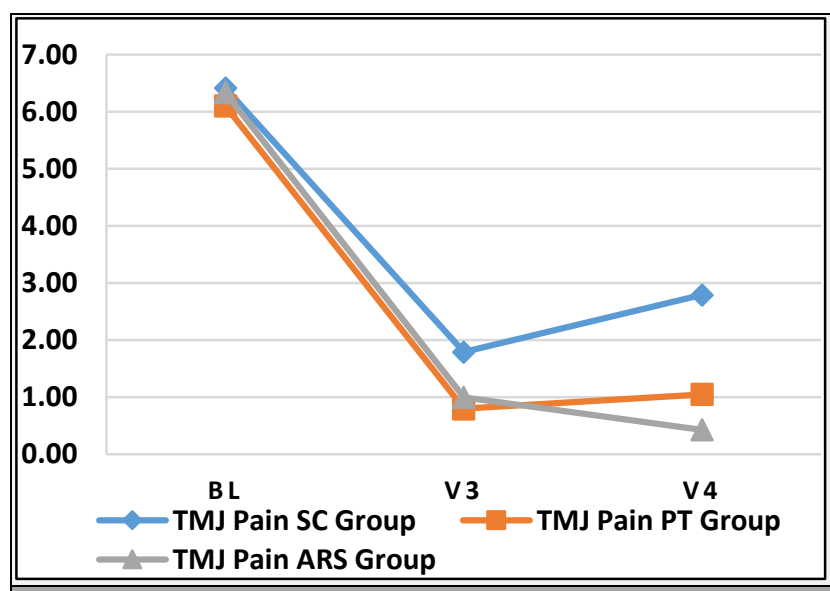

Figure 2. TMJ Pain, SC: Self-Care Group, PT: Physiotherapy Group, ARS: Anterior Repositioning Splint. BL: Baseline, V3: Fourth Week Follow-Up (End of Treatment), V4: Sixteenth Week Follow-Up (Post-Treatment)

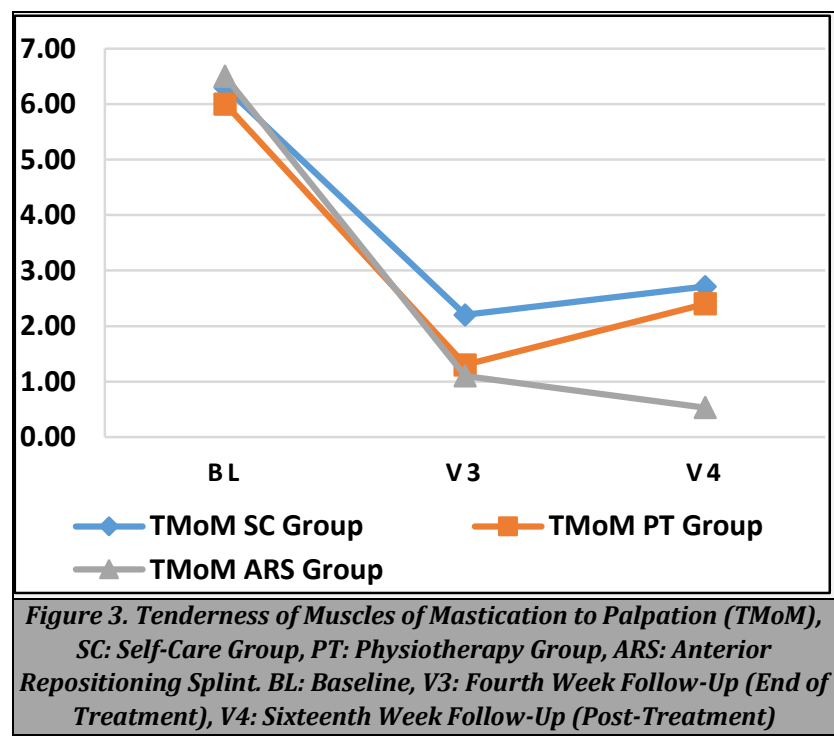



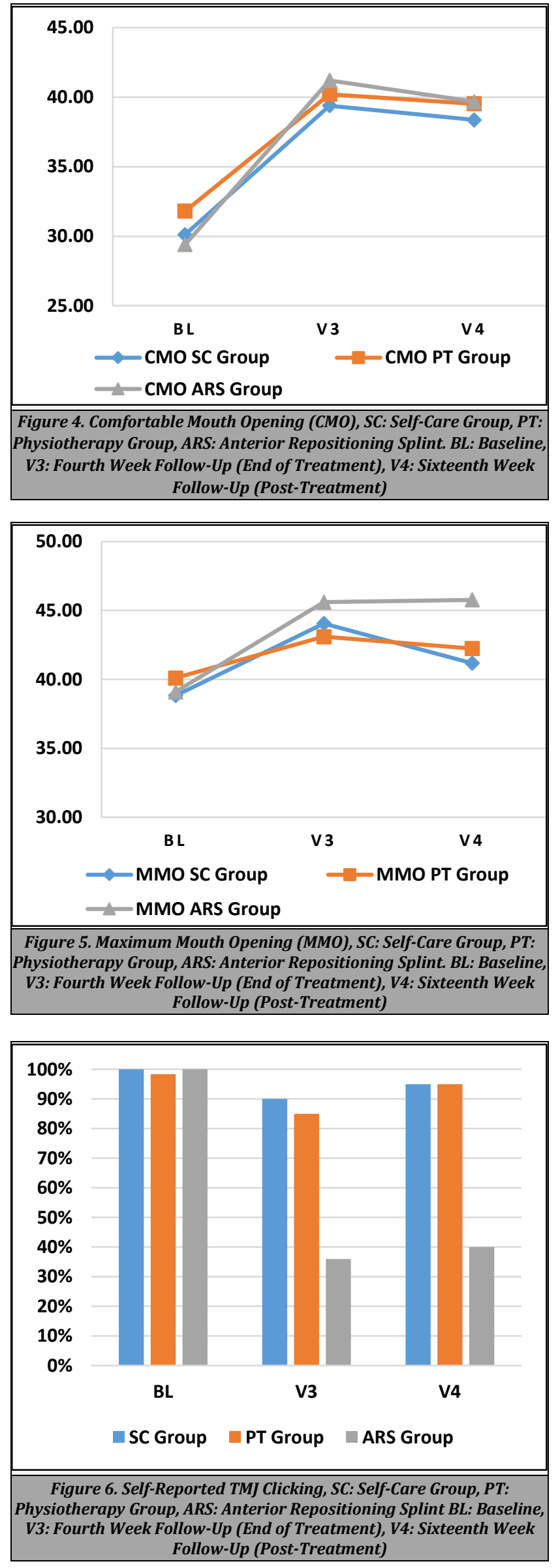

\begin{tabular}{|ccccc|}
\hline Demographic features & $\begin{array}{c}\text { SC Group } \\
\mathbf{n = 6 3}\end{array}$ & $\begin{array}{c}\text { PT Group } \\
\mathbf{n = 6 3}\end{array}$ & $\begin{array}{c}\text { ARS Group } \\
\mathbf{n = 6 3}\end{array}$ & $\mathbf{P}$ \\
Age (Mean $\left.\pm \mathrm{SD}^{*}\right)$ & $35 \pm 14$ & $34 \pm 14$ & $36 \pm 12$ & NS \\
Male \% & 38 & 38 & 40 & NS \\
Female \% & 61.8 & 61.9 & 60 & NS \\
$\begin{array}{c}\text { Number of year with TMD } \\
\text { (Mean } \pm \text { SD*) }^{*}\end{array}$ & $6 \pm 9$ & $5 \pm 6$ & $5 \pm 5$ & NS \\
Prior therapy for TMDs (\%) & 21 & 22 & 19 & NS \\
\hline Table 1. Baseline demographics of the treatment groups \\
SD*: Standard Deviation, NS: Not Significant \\
\hline
\end{tabular}

\begin{tabular}{|c|c|c|c|c|c|}
\hline $\begin{array}{c}\text { Clinical ExaminationF } \\
\text { Finding }\end{array}$ & $\begin{array}{l}\text { ollow } \\
\text {-up }\end{array}$ & $\begin{array}{c}\text { SC Group } \\
n=61\end{array}$ & $\begin{array}{l}\text { PT Group } \\
n=63\end{array}$ & $\begin{array}{c}\text { ARS Group } \\
n=63\end{array}$ & $\mathbf{P}$ \\
\hline \multirow{3}{*}{$\begin{array}{l}\text { TMJ pain on palpation } \\
\quad\left(\text { Mean } \pm \mathrm{SD}^{*}\right)\end{array}$} & BL & $6.2 \pm 1.2$ & $6.1 \pm 1.6$ & $6.33 \pm 1.4$ & 0.81 \\
\hline & V3 & $1.7 \pm 0.9$ & $0.8 \pm 1.0$ & $1.0 \pm 1.5$ & 0.84 \\
\hline & V4 & 06 & 1.10 & $0.43 \pm 0.57$ & 0.56 \\
\hline \multirow{3}{*}{$\begin{array}{c}\text { Muscle of mastication } \\
\text { pain on palpation (Mean } \\
\pm \text { SD) }\end{array}$} & BL & 4 & & & 0.81 \\
\hline & V3 & & & & 0.79 \\
\hline & V4 & $2.71 \pm 1$ & $2.40 \pm 1.68$ & $0.53 \pm 1.22$ & 0.72 \\
\hline \multirow{3}{*}{$\begin{array}{c}\text { Comfortable mouth } \\
\text { opening (CMO) in } \\
\text { millimeter (Mean } \pm \text { SD*) }\end{array}$} & BL & & 2 & 29.4 & 0.96 \\
\hline & v3 & 01 & 2 & $41.2 \pm 1.0$ & 0.01 * \\
\hline & v4 & $38.35 \pm 1.35$ & $39.52 \pm 1.29$ & $39.67 \pm 1.47$ & 0.07 \\
\hline \multirow{3}{*}{$\begin{array}{l}\text { Maximum mouth opening } \\
\text { (MMO) in millimeter } \\
\text { (Mean } \pm \mathrm{SD}^{*} \text { ) }\end{array}$} & BL & $38.8 \pm 1.1$ & $40.1 \pm 1.0$ & $39.1 \pm 1.3$ & 0.96 \\
\hline & v3 & $43.4 \pm 1.4$ & $43.1 \pm 1.4$ & $45.6 \pm 1.6$ & $0.02 *$ \\
\hline & v4 & $42.26 \pm 1.69$ & $42.24 \pm 1.59$ & $45.77 \pm 0.21$ & $0.04^{*}$ \\
\hline \multirow{3}{*}{ TMJ clicking (\%) } & & & & & 0.93 \\
\hline & V3 & & & & $0.03 *$ \\
\hline & V4 & $94.1 \%$ & $95.2 \%$ & $40.1 \%$ & $0.04 *$ \\
\hline
\end{tabular}

Table 2. Treatment Outcomes (Clinical Examination Findings), At Three Different Point of Time For The Three Trial Groups (ANOVA and Tukey Test). Baseline (BL), End of Treatment or Fourth Week FollowUp (V3) and Sixteen Weeks Post Treatment Follow-Up (V4), *: Statistically Significant.

\section{DISCUSSION}

We had initially hypothesised that short-term benefits of PT (four weeks from BL) and ARS (three months from BL) groups would be more than SC group and the long-term benefits (16 weeks / 4 month from BL) would be equal in the three treatment (SC, PT and ARS) groups. Our study results indicated that, the short term benefits were comparable in all three groups, except CMO, MMO and TMJ clicking (Table 2) was more pronounced in ARS group. However, the long term (16 weeks / 4 month from BL) benefits did not show statistically significant difference between the treatments groups in the outcomes measured, except MMO and TMJ clicking. Based on the aforementioned outcomes the following were our key findings: 1) all three management strategies were successful in ameliorating the pain intensity reported with DDwR; 2) patients wearing AR Splint with selfmanagement and those undergoing physiotherapy plus selfmanagement reported faster significant improvements compared to the control group (self-management only). Thus emphasising the importance of occlusal splints and physiotherapy in the management of DDwR; 3) there was no improvement of TMJ sound (clicking) in patients of PT and SC group. The aforementioned findings led us to reject the null hypothesis.

Prior studies have shown that splint and physiotherapy therapy can successfully reduce the signs and symptoms associated with TMDs including DDwR.9,22,23,17,24,25,26,27,28,29 However, our RCT data (Table 2) indicated a significant difference in only two (MMO and TMJ clicking) of the five treatment outcomes measured. Therefore, our results are not in full agreement with the aforementioned studies, one 
reasons for this difference could be the RCTs that supported the effectiveness of splint therapy had employed a non-contact control splint 17,25 and stabilising type of splint ${ }^{25}$ as the control group, while we used self-management as the control. Other factors that could have influenced this outcome are 1) the benign natural course of the disease, ${ }^{30} 2$ ) the placebo effect and 3) regression towards the mean. It is noteworthy that our findings are only in partial agreement with many other RCTs $17,31,32,12,14,15,16,18,33$ and systematic reviews ${ }^{34,35}$ that reported no superiority of splint therapy over other treatment approaches, because we did observe a significant improvement in MMO and TMJ clicking. However, the aforementioned studies used jaw self-exercise ${ }^{17}$ and patient education $^{18}$ as a control. Therefore, we believe the inconsistency between our study and others may be attributable to differences in control groups.

Intra group analysis revealed the improvement in outcomes measured were faster / earlier in PT and ARS group. The early improvement in DDwR symptoms in PT group can be attributed to the therapeutic effects induced by the ultra sound waves; 1 ) the creation of acoustic vibrations causing movement of the cell membrane, fluid and macromolecules, ${ }^{36}$ 2) The mechanical stimulation that subsequently change the physical and biological properties of the cells, (cell membrane permeability, fluid movement and exchange of intracellular and extracellular ions) that eventually alter cell growth and metabolism. ${ }^{37}$ The early improvement noticed in AR split wearing group could be due to the new intraoral condition, which is designed to interfere with the impact of certain trigeminal neural circuits. ${ }^{38}$ Except ARS group no other treatment group showed significant improvement or reduction of TMJ clicking, however, the improvement in DDwR symptoms (except TMJ clicking) noticed in SC group could be due to the natural and progressive adaptation of TMJ structures to loading regardless if the patients received US therapy or wore AR splint or otherwise ${ }^{30}$ and / or due to morphological alterations in the TMJ disc itself, that could eliminate the physical barrier for jaw translation and thus decrease the joint sound. ${ }^{39}$

The superiority of US therapy or AR splint therapy over self-care was not demonstrated in our study results. On the contrary we observed almost same pattern of improvement in all three treatment groups. These findings suggest that the appropriateness and necessity of US therapy or AR splint therapy as an initial choice of treatment for DDwR is limited. Similar results have been reported by recent putative studies. ${ }^{31,12}$ Likewise, other form of non-surgical treatment such as transcutaneous electrical nerve stimulation, acupuncture, behavioural changes, relaxation training and biofeedback have also demonstrated their usefulness in the management of TMD signs and symptoms. 10,23,24,40 Furthermore, long term use of AR splint (especially 24 hours a day) could lead to a permanent mandibular anterior positioning and a posterior open bite. The fact that, many cases of DDwR are self-limiting $23,30,41$ should not discourage clinicians from initiating prompt intervention as it contributes to a more rapid improvement in quality of life of TMD patients. ${ }^{24}$

\section{Limitations}

The following limitations should be borne in mind while interpreting the presented results; first possible limitation was selection bias, as some of the participants of our study were referred from general practice who may have had undergone unsuccessful TMD management therapies in the past. Therefore, inclusion of these study subjects could have affected our results. However, the study subjects were randomly allocated to one of the treatment groups and we also included a wash out period of two weeks before the patients were recruited into the trial to overcome this limitation.

The second limitation; we were unable to include a notreatment group in our study, because, a majority of patients with DDwR indicated a desire for positive treatment while the study was explained. Therefore, we eliminated the inclusion of a no-treatment group in our study. Thus, our results can only estimate the combine effect of physio and splint with self-care therapy rather than the single effect.

The third limitation could be; while the post graduate students involved in collecting the treatment outcome data were blinded to the treatment groups, it was impossible to blind the clinicians treating the patients and to determine whether they inadvertently would have modified their treatment approach.

Our study inclusion criteria was restricted to RDC / TMD Axis I ("psychosocially functional") subjects only, therefore, we were unable to determine whether people with DDwR associated with psychosocial dysfunction would have had the similar outcomes.

\section{CONCLUSIONS}

Individually all the three treatment modalities were effective in alleviating the signs and symptoms of DDwR but did not differ from each other in their long-term benefits. However, almost equivalent improvement noted across the three groups is encouraging in terms of the clinical implications and difference in cost and chair time required for the three different treatment modalities used in our study (i.e. less amount of provider time was spent on SC group, whereas, additional time, and costs were incurred in providing US therapy or AR splint therapy).

TMDs are known to be multifactorial, likewise, patients' response to therapy is also a complex interaction between patient, dentist, and the treatment, rather than a simple causeeffect phenomenon. Hence, we believe that comprehensive care using a combination therapy represents a more suitable and practical primary treatment choice of DDwR. Additionally, cost effective and time saving treatment options can contribute towards rapid improvement of patient's quality of life. Thus, multimodal therapy (comprehensive care) rather than monotherapy (physiotherapy / splint therapy alone) could be recommended to treat DDwR. Further, large sample studies are required with long-term follow-up with inclusion of other treatment modalities such as counselling, occlusal correction along with splint therapy, pharmacotherapy, and physiotherapy.

Data sharing statement provided by the authors is available with the full text of this article at jemds.com.

Financial or other competing interests: None.

Disclosure forms provided by the authors are available with the full text of this article at jemds.com.

We thank the clinicians and post graduate students of Department of Oral Medicine and Radiology, in Government Dental College, 
Bangalore, who took part in the screening and follow - up examination of the study patients. We thank the physiotherapist in the Department of Physiotherapy, Victoria Hospital, Bangalore, for providing physiotherapy to the patients. We thank the staff and post graduate students of the Department of Prosthodontics, Government Dental College, Bangalore, for their assistance in fabricating the anterior repositioning splints. We also thank Mr. Jagannath for the statistical guidance and assistance.

The protocol for this trial was approved by the research ethics committee of Government Dental College, Bangalore, Karnataka, India. All patients signed the written informed consent to take part in the trial.

\section{REFERENCES}

[1] De Leeuw R, Klasser G. Orofacial pain: guidelines for assessment, diagnosis, and management. $5^{\text {th }}$ edn. Chicago: Quintessence Publishing Co. Inc 2013.

[2] Schiffman EL, Fricton JR, Haley DP, et al. The prevalence and treatment needs of subjects with temporomandibular disorders. J Am Dent Assoc 1990;120(3):295-303.

[3] Schiffman E, Ohrbach R, Truelove E, et al. Diagnostic criteria for temporomandibular disorders (DC/TMD) for clinical and research applications: recommendations of the International RDC/TMD Consortium Network and Orofacial Pain Special Interest Group. J Oral Facial Pain Headache 2014;28(1):6-27.

[4] Conti PC, Ferreira PM, Pegoraro LF, et al. A cross-sectional study of prevalence and etiology of signs and symptoms of temporomandibular disorders in high school and university students. J Orofac Pain 1996;10(3):254-62.

[5] Grosfeld 0, Jackowska M, Garnecka B. Results of epidemiological examination of the temporomandibular joint in adolescents and young adults 1985;12(2):95-105.

[6] Hiltunen K. Schmidt-Kaunisaho K, Nevalainen J, et al. Prevalence of signs of temporomandibular disorders among elderly inhabitants or Helsinki, Finland. Acta Odontol Scand 1995;53(1):20-3.

[7] Lundh H, Westesson PL, Kopp S, et al. Anterior repositioning splint in the treatment of temporomandibular joints with reciprocal clicking: comparison with a flat occlusal splint and an untreated control group. Oral Surg Oral Med Oral Pathol 1985;60(2):131-6.

[8] Di Fabio RP. Physical therapy for patients with TMD: a descriptive study of treatment, disability, and health status. J Orofac Pain 1998;12(2):124-35.

[9] Gray RJ, Quayle AA, Hall CA, et al. Physiotherapy in the treatment of temporomandibular joint disorders: a comparative study of four treatment methods. Br Dent J 1994;176(7):257-61.

[10] Linde C, Isacsson G, Jonsson BG. Outcome of 6-week treatment with transcutaneous electric nerve stimulation compared with splint on symptomatic temporomandibular joint disk displacement without reduction. Acta Odontol Scand 1995;53(2):92-8.

[11] Murakami K, Hosaka H, Moriya Y, et al. Short-term treatment outcome study for the management of temporomandibular joint closed lock. A comparison of arthrocentesis to nonsurgical therapy and arthroscopic lysis and lavage. Oral Surg Oral Med Oral Pathol Oral Radiol Endod 1995;80(3):253-7.
[12] Truelove E, Huggins KH, Mancl L, et al. The efficacy of traditional, low-cost and nonsplint therapies for temporomandibular disorder: a randomized controlled trial. J Am Dent Assoc 2006;137(8):1099-107.

[13] Niemelä K, Korpela M, Raustia A, et al. Efficacy of stabilisation splint treatment on temporomandibular disorders. J Oral Rehabil 2012;39(11):799-804.

[14] Ficnar T, Middelberg C, Rademacher B, et al. Evaluation of the effectiveness of a semi-finished occlusal appliance--a randomized, controlled clinical trial. Head Face Med 2013;9:5.

[15] Magnusson T, Syren M. Therapeutic jaw exercises and interocclusal appliance therapy. A comparison between two common treatments of temporomandibular disorders. Swed Dent J 1999;23(1):27-37.

[16] Glaros AG, Kim-Weroha N, Lausten L, et al. Comparison of habit reversal and a behaviorally-modified dental treatment for temporomandibular disorders: a pilot investigation. Appl Psychophysiol Biofeedback 2007;32(3-4):149-54.

[17] Haketa T, Kino K, Sugisaki M, et al. Randomized clinical trial of treatment for TMJ disc displacement. J Dent Res 2010;89(11):1259-63.

[18] Michelotti A, Iodice G, Vollaro S, et al. Evaluation of the short-term effectiveness of education versus an occlusal splint for the treatment of myofascial pain of the jaw muscles. J Am Dent Assoc 2012;143(1):47-53.

[19] Dworkin SF, LeResche L. Research diagnostic criteria for temporomandibular disorders: review, criteria, examinations and specifications, critique. J Craniomandib Disord 1992;6(4):301-55.

[20] Schiffman EL, Truelove EL, Ohrbach R, et al. The Research diagnostic criteria for temporomandibular disorders. I: overview and methodology for assessment of validity. J Orofac Pain 2010;24(1):7-24.

[21] Look JO, Schiffman EL, Truelove EL, et al. Reliability and validity of Axis I of the Research diagnostic criteria for temporomandibular disorders (RDC/TMD) with proposed revisions. J Oral Rehabil 2010;37(10):744-59.

[22] Al-Baghdadi M, Durham J, Araujo-Soares V, et al. TMJ disc displacement without reduction management: a systematic review. J Dent Res 2014;93(7 Suppl):37S-51S.

[23] Conti PCR, de Alencar EN, da Mota Correa AS, et al. Behavioural changes and occlusal splints are effective in the management of masticatory myofascial pain: a shortterm evaluation. J Oral Rehabil 2012;39(10):754-60.

[24] Medlicott MS, Harris SR. A systematic review of the effectiveness of exercise, manual therapy, electrotherapy, relaxation training, and biofeedback in the management of temporomandibular disorder. Phys Ther 2006;86(7):955-73.

[25] Cleland J, Palmer J. Effectiveness of manual physical therapy, therapeutic exercise, and patient education on bilateral disc displacement without reduction of the temporomandibular joint: a single-case design. J Orthop Sports Phys Ther 2004;34(9):535-48.

[26] Kuttila M, Bell YL, Savolainen-Niemi E, et al. Efficiency of occlusal appliance therapy in secondary otalgia and temporomandibular disorders. Acta Odontol Scand 2002;60(4):248-54.

[27] Ekberg E, Nilner M. Treatment outcome of appliance therapy in temporomandibular disorder patients with 
myofascial pain after 6 and 12 months. Acta Odontol Scand 2004;62(6):343-9.

[28] de Felício CM, de Oliveira MM, da Silva MAMR. Effects of orofacial myofunctional therapy on temporomandibular disorders. Cranio 2010;28(4):249-59.

[29] Turner JA, Mancl L, Aaron LA. Short-and long-term efficacy of brief cognitive-behavioral therapy for patients with chronic temporomandibular disorder pain: a randomized, controlled trial. Pain 2006;121(3):181-94.

[30] Conti PCR, Miranda JES, Conti ACCF, et al. Partial time use of anterior repositioning splints in the management of TMJ pain and dysfunction: a one-year controlled study. J Appl Oral Sci 2005;13(4):345-50.

[31] Conti PCR, da Mota Corrêa AS, Lauris JRP, et al. Management of painful temporomandibular joint clicking with different intraoral devices and counseling: a controlled study. J Appl Oral Sci 2015;23(5):529-35.

[32] van der Glas HW, Buchner R, van Grootel RJ. Comparison of treatment options for myogenous temporomandibular dysfunction. Ned Tijdschr Tandheelkd 2000;107(12):505-12.

[33] Nagata K, Maruyama H, Mizuhashi R, et al. Efficacy of stabilisation splint therapy combined with non-splint multimodal therapy for treating RDC/TMD axis I patients: a randomised controlled trial. J Oral Rehabil 2015;42(12):890-9.

[34] Al-Ani MZ, Davies SJ, Gray RJM, et al. Stabilisation splint therapy for temporomandibular pain dysfunction syndrome. Cochrane Database Syst Rev 2004;(1):CD002778.

[35] Forssell H, Kalso E. Application of principles of evidencebased medicine to occlusal treatment for temporomandibular disorders: are there lessons to be learned? J Orofac Pain 2004;18(1):9-22.

[36] Kim Y, Rhim H, Choi MJ, et al. High-intensity focused ultrasound therapy: an overview for radiologists. Korean J Radiol 2008;9(4):291-302.

[37] ter Haar G. Therapeutic applications of ultrasound. Prog Biophys Mol Biol 2007;93(1-3):111-29.

[38] Klasser GD, Greene CS. Oral appliances in the management of temporomandibular disorders. Oral Surg Oral Med Oral Pathol Oral Radiol Endod 2009;107(2):212-23.

[39] Conti PCR, dos Santos CN, Kogawa EM, et al. The treatment of painful temporomandibular joint clicking with oral splints: a randomized clinical trial. J Am Dent Assoc 2006;137(8):1108-14.

[40] Bijjaragi S, Majid IA, Saraswathi FK, et al. Pain management in temporomandibular joint disorders by active and placebo transcutaneous electric nerve stimulation: a comparative study. Am J Drug Deliv Ther 2015;2(1):20-8.

[41] de Leeuw R, Boering G, Stegenga B, et al. Symptoms of temporomandibular joint osteoarthrosis and internal derangement 30 years after non-surgical treatment. Cranio 1995;13(2):81-8. 